\title{
30 Jahre nach der Wiedervereinigung hat das Bruttoinlandsprodukt als Indikator ausgedient
}

\author{
Der Vergleich der Lebensverhältnisse in Ost- und Westdeutschland orientiert sich häufig am \\ Bruttoinlandsprodukt je Einwohner:in. Dieses Maß ist jedoch für sich genommen kein guter \\ Wohlfahrtsindikator. Es ist davon auszugehen, dass gemessen am Bruttoinlandsprodukt \\ je Einwohner:in in absehbarer Zukunft keine wesentliche weitere Angleichung der \\ Wirtschaftskraft in Ost- und Westdeutschland mehr stattfinden wird. Denn die Altersstruktur \\ Ostdeutschlands, d.h. das Verhältnis von Erwerbstätigenzahl zu Einwohnerzahl, ist \\ ungünstiger als im Westen. Betrachtet man hingegen wichtige Wohlfahrtsindikatoren wie \\ Konsummöglichkeiten, Lebenserwartung, Freizeit und Einkommensungleichheit, so sind die \\ Lebensverhältnisse in Ost- und Westdeutschland ähnlicher als das Bruttoinlandsprodukt je \\ Einwohner:in suggeriert. In den Debatten über den Aufholprozess Ostdeutschlands sollte \\ daher stärker auf die Arbeitsproduktivität als Maß für die Wirtschaftskraft und auf andere \\ Wohlfahrtsindikatoren als Maß für die Angleichung der Lebensverhältnisse abgestellt werden.
}

Seit vielen Jahren erscheint im Vorfeld des 3. Oktober der Jahresbericht der Bundesregierung zum Stand der Deutschen Einheit (BMWi, 2020). Im Jahresbericht 2020 wird erneut das Bruttoinlandsprodukt (BIP) je Einwohner:in als Indikator zum Stand des wirtschaftlichen Aufholprozesses herausgehoben. Die ostdeutschen Flächenländer kamen 2019 auf knapp $73 \%$ in Relation zum Bundesdurchschnitt. Angesichts des Ausgangsniveaus von $37 \%$ im Jahr 1991 ist das ein beachtlicher Fortschritt. Aber wie hilfreich ist dieser Indikator? Ist er ein sinnvoller Gradmesser für die Angleichung der Lebensverhältnisse, die sich die Politik als Ziel setzt? Vermutlich ist er das eher nicht. Im Gegenteil - das Festhalten an diesem Indikator ist eher dazu geeignet, für weitere Enttäuschungen bei vielen Menschen zu sorgen. Denn implizit wird mit diesem Maß die Erwartung geschürt, dass $100 \%$ die Zielmarke seien und dass es nur eine Frage der Zeit sei, wann die $100 \%$ erreicht werden. Mehrere Gründe sprechen jedoch dagegen, dass dies ein realistisches und angemessenes Ziel ist. Zum einen ist eine vollständige Angleichung vor dem Hintergrund historischer Erfahrungen nicht zu erwarten, und zum anderen trägt der demografische Wandel dazu bei, dass das Verhältnis von Erwerbstätigen zur Gesamtbevölkerung in Ostdeutschland niedriger ist und bleibt als in Westdeutschland, was für sich genommen die Lücke beim BIP pro Kopf vergrößert.

(c) Der/die Autor:in(nen) 2021. Open Access: Dieser Artikel wird unter der Creative Commons Namensnennung 4.0 International Lizenz veröffentlicht (creativecommons.org/licenses/by/4.0/deed.de).

Open-Access wird durch die ZBW - Leibniz-Informationszentrum Wirtschaft gefördert.

\section{Historische Maßstäbe}

Historische Vergleiche bezüglich der regionalen wirtschaftlichen Leistungsfähigkeit sind in Deutschland schwierig, weil sich die Gebietsabgrenzungen in den vergangenen einhundert Jahren mehrfach geändert haben. Wirtschaftshistoriker:innen haben jedoch Rückschätzungen des BIPs und der Einwohnerzahl für die heutige administrative Abgrenzung der Regionen in Europa (NUTS-2) vorgenommen (Roses und Wolf, 2019). Aggregiert man die deutschen NUTS-2-Regionen zu Westdeutschland, Ostdeutschland (ohne Berlin) und Berlin, so ergibt sich folgendes Bild (vgl. Abbildung 1): Bis zum Zweiten Weltkrieg war die Wirtschaftskraft in Ost- und Westdeutschland in etwa gleich groß, in der Hauptstadt Berlin lag sie über dem Durchschnitt. Nach dem Zweiten Weltkrieg nahm die Wirtschaftskraft in Westdeutschland schneller zu als in Ostdeutschland; zwischen 1980 und 1990 ging das BIP je Einwohner:in in Ostdeutschland sogar zurück. Nach der Wiedervereinigung stieg dieser Wert in den 1990er Jahren in Ostdeutschland und in Berlin schneller als in Westdeutschland, d.h. die Wirtschaftskraft näherte

Prof. Dr. Oliver Holtemöller ist stellvertretender Präsident und leitet die Abteilung Makroökonomik am Leibniz-Institut für Wirtschaftsforschung Halle (IWH). 
Abbildung 1

Langfristige Entwicklung der Wirtschaftskraft in Deutschland

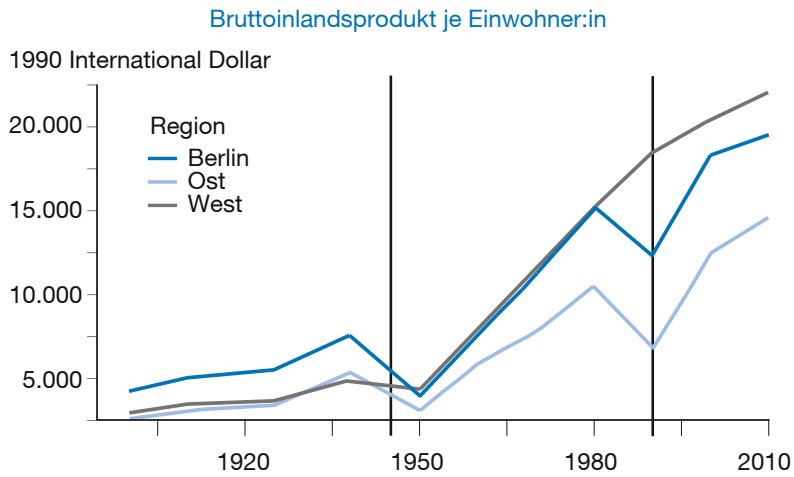

Anmerkung: Ostdeutschland ohne Berlin. Vertikale Linien 1945 und 1990.

Quelle: Roses und Wolf (2019), eigene Berechnungen und Darstellung.

sich an. Die Annäherungsgeschwindigkeit hat sich seitdem jedoch erheblich verlangsamt.

Die Tatsache, dass das BIP je Einwohner:in in Ost- und Westdeutschland vor dem Zweiten Weltkrieg in etwa gleich hoch war, sollte nicht darüber hinwegtäuschen, dass sich dahinter eine erhebliche regionale Spreizung verbarg. So liegt das BIP pro Kopf etwa in Brandenburg oder Mecklenburg-Vorpommern nicht erst seit der deutschen Teilung, sondern schon weitaus länger unter dem Durchschnitt (vgl. Abbildung 2). Allgemein lassen sich die heute zu beobachtenden ökonomischen Unterschiede nicht nur auf die deutsche Teilung und die Zentralverwaltungswirtschaft zu DDR-Zeiten zurückführen, sondern waren teils schon deutlich früher sichtbar (Becker et al., 2020).

Auch international ist zu beobachten, dass Unterschiede in der wirtschaftlichen Leistungsfähigkeit überwiegend sehr persistent sind. Vielerorts findet zwar ein Aufholen von niedrigem auf mittleres Niveau statt. Aber eine weitgehende Angleichung der internationalen wirtschaftlichen Leistungsfähigkeit findet weder zwischen den Staaten noch zwischen den Regionen innerhalb der meisten Staaten statt. Vor diesem Hintergrund ist nicht zu erwarten, dass sich das BIP je Einwohner:in in Deutschland noch sehr viel weiter angleichen wird.

\section{Konvergenz der Produktivität und Divergenz des BIP je Einwohner:in}

Die Konvergenz des regionalen BIP pro Kopf wird auch dadurch beeinflusst, dass Wanderungen zwischen den Regionen stattfinden. Es besteht insbesondere für jun-

Abbildung 2

Langfristige Entwicklung der Wirtschaftskraft in den deutschen Regionen (1900 bis 2019)
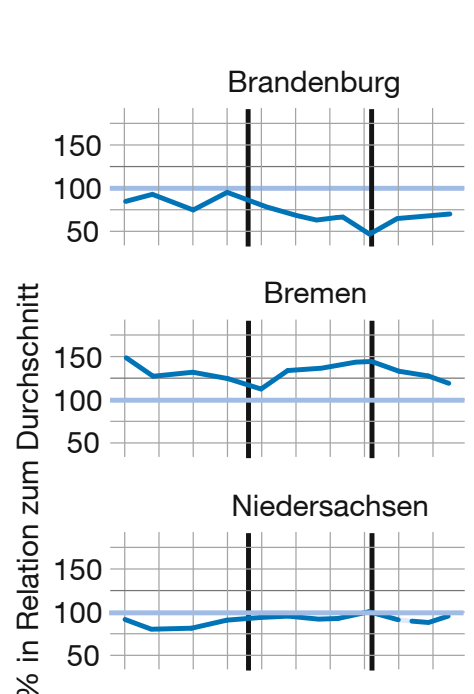

১ீ

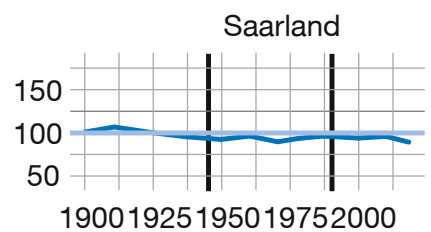

Bruttoinlandsprodukt je Einwohner:in
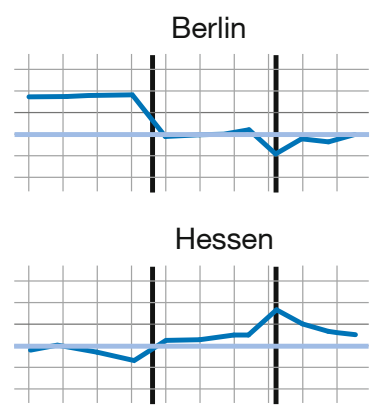

Nordrhein-Westfalen

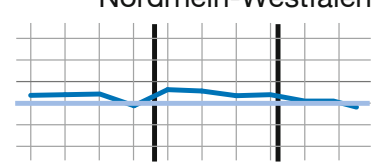

Sachsen

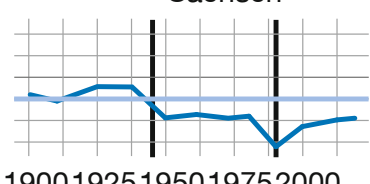

19001925195019752000

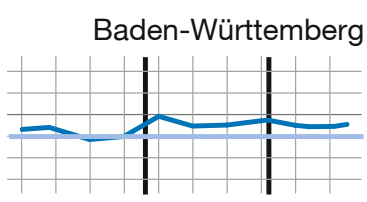

Hamburg

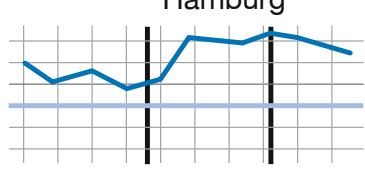

Rheinland-Pfalz

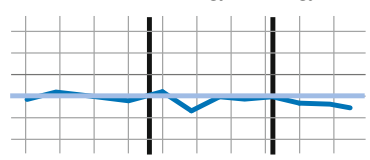

Sachsen-Anhalt

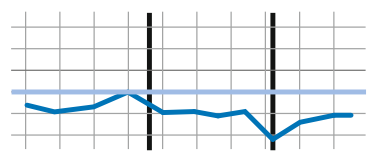

19001925195019752000

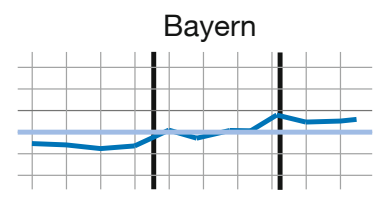

Mecklenburg-Vorpommern

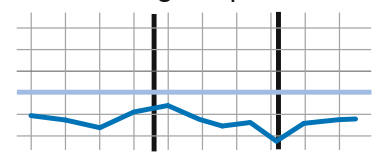

Schleswig-Holstein

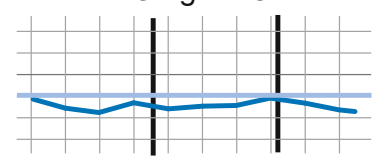

Thüringen

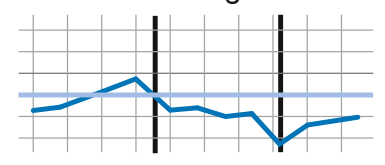

19001925195019752000

Anmerkung: Vertikale Linien 1945 und 1990.

Quellen: 1900 bis 2010: Roses und Wolf (2019); 2019: VGR der Länder, eigene Berechnungen und eigene Darstellung. 
ge, gut qualifizierte Erwerbstätige ein Anreiz, in Regionen abzuwandern, in denen die durchschnittliche Arbeitsproduktivität und damit das Reallohnniveau höher ausfallen als in ihrer Heimatregion. Dadurch steigen sowohl ihr individuelles Einkommen als auch das aggregierte Einkommen von Heimat- und Zielregion. In der Tat war die Migration von Ostdeutschland nach Westdeutschland ein wesentlicher Faktor des Anpassungsprozesses nach der Wiedervereinigung (Lindner, 2017; Wieschemeyer, 2017). Dies führte aber auch dazu, dass sich das Verhältnis der Erwerbspersonenzahl zur Zahl der Bevölkerung insgesamt in Ostdeutschland schneller verringert hat als in Westdeutschland.

Über den unmittelbaren Effekt der Abwanderung von Personen im erwerbsfähigen Alter auf die Altersstruktur hinaus verstärkt sich der Effekt langfristig durch weniger Geburten. Dementsprechend fallen die Bevölkerungsvorausberechnungen für Ostdeutschland ungünstiger aus als für Westdeutschland. So wird die Zahl der 67-Jährigen und Älteren in Relation zu den 20- bis unter 67-Jährigen gemäß mittlerem Szenario in Westdeutschland von 29,7\% im Jahr 2018 auf 49,8\% im Jahr 2060 und in Ostdeutschland im gleichen Zeitraum von 36,9\% auf 57,3\% zunehmen (Statistisches Bundesamt, 2019).

Das BIP je Einwohner:in kann wie folgt zerlegt werden:

$$
\frac{B I P}{E W}=\frac{B I P}{E T} \times \frac{E T}{E W},
$$

wobei EW die Einwohnerzahl und ET die Zahl der Erwerbstätigen darstellt. Das Verhältnis ET/EW hängt erheblich von dem Verhältnis der Zahl der über 67-Jährigen zu der Zahl der 20- bis unter 67-Jährigen ab. Selbst wenn sich die Arbeitsproduktivität BIP/ET in Ost- und Westdeutschland weiter annähern sollte, dürfte ein deutlicher Unterschied beim BIP pro Kopf bleiben, weil die Lücke beim Verhältnis ET/EW sich eher weiter öffnen als schlieBen dürfte (Holtemöller und Irrek, 2012).

\section{Lebensverhältnisse in Ost und West ähnlicher als die Wirtschaftskraft}

Das BIP je Einwohner:in ist zwar ein relevantes Maß für die Wirtschaftskraft einer Region, aber es ist für sich genommen kein gutes Wohlfahrtsmaß. Das tatsächliche Wohlbefinden der Bevölkerung lässt sich anhand anderer Indikatoren besser approximieren. So schlagen etwa Jones und Klenow (2016) vor, die Lebenserwartung, den privaten Konsum pro Kopf, die Freizeit und die Ungleichheit als Kriterien heranzuziehen. Diese Indikatoren weisen viel geringere Unterschiede zwischen Ost- und Westdeutschland auf als das BIP pro Kopf (vgl. Abbildung 3).
Abbildung 3

Wohlfahrtsindikatoren
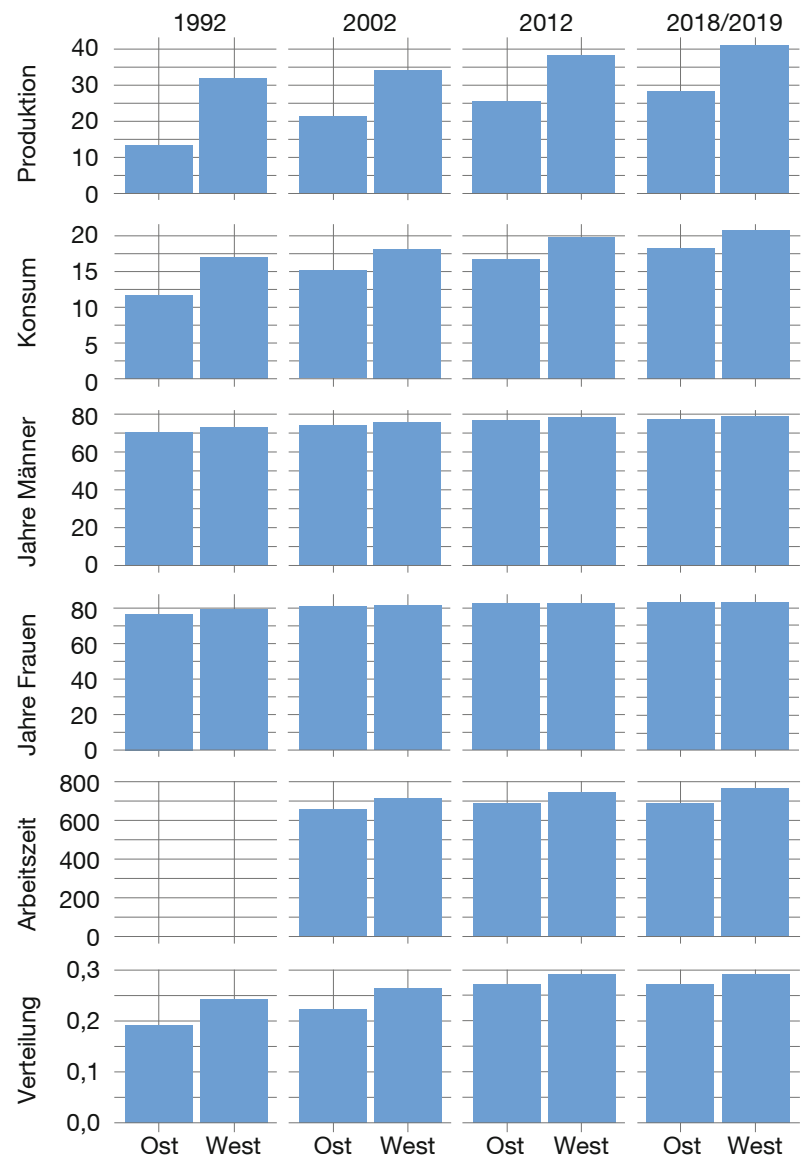

Anmerkungen: Produktion = Bruttoinlandprodukt je Einwohner:in in 1.000 Euro in Preisen von 2015 (letzter Wert 2019); Konsum = Private Konsumausgaben je Einwohner:in in 1.000 Euro in Preisen von 2015 (letzter Wert 2018); Jahre Männer = Lebenserwartung eines neugeborenen Jungen in Jahren (letzter Wert Dreijahresdurchschnitt 2017-2019); Jahre Frauen = Lebenserwartung eines neugeborenen Mädchens in Jahren (letzter Wert Dreijahresdurchschnitt 2017-2019), Arbeitszeit = Arbeitsstunden je Einwohner:in (letzter Wert: 2019); Verteilung = Gini-Koeffizient des Äquivalenzeinkommens (letzter Wert: 2019).

Quellen: Produktion, Konsum und Arbeitszeit: Volkswirtschaftliche Gesamtrechnungen der Länder; Lebenserwartung: Statistisches Bundesamt; Verteilung: Goebel et al. (2009) für 1992 und 2002, Statistisches Bundesamt für 2012 und 2019.

Während die Ost-West-Lücke beim BIP je Einwohner:in in Ostdeutschland im Jahr 2019 bei $32 \%$ lag, waren es bei den Konsumausgaben pro Kopf nur noch $12 \%$, vor allem aufgrund von Transferleistungen über das Sozialversicherungssystem. Die Lebenserwartung ostdeutscher Frauen hat ihren Rückstand gegenüber westdeutschen Frauen, der 1992 noch gut zwei Jahre betrug, komplett aufgeholt; bei Männern ist der Abstand von mehr als drei Jahren auf gut ein Jahr zurückgegangen. Bei den Indikatoren Freizeit und Verteilung liegt Ostdeutschland vorne. Während 2019 in Westdeutschland 765 Arbeitsstunden je Einwohner:in 
anfielen, waren es in Ostdeutschland 688, also gut $10 \%$ weniger (hier spielt die Altersstruktur wieder eine Rolle). Der Gini-Koeffizient der Äquivalenzeinkommen als Maß für die Einkommensungleichheit innerhalb der Regionen hat in Ostdeutschland seit 1992 deutlich zugenommen, liegt aber immer noch unter dem westdeutschen Niveau, d.h. die Einkommensungleichheit ist in Westdeutschland etwas stärker als in Ostdeutschland. Alles in allem zeigt sich, dass das BIP je Einwohner:in die Angleichung der Lebensverhältnisse in Ost- und Westdeutschland nur unzureichend widerspiegelt.

\section{Fazit}

Der Vergleich der Lebensverhältnisse in Ost- und Westdeutschland orientiert sich häufig am BIP je Einwohner:in. Dieses $M a ß$ ist jedoch für sich genommen kein guter Wohlfahrtsindikator. Es ist davon auszugehen, dass gemessen am BIP pro Kopf in absehbarer Zukunft keine wesentliche weitere Angleichung der Wirtschaftskraft in Ostund Westdeutschland mehr stattfinden wird. Denn die Altersstruktur Ostdeutschlands, d.h. das Verhältnis von Erwerbstätigenzahl zu Einwohnerzahl, ist ungünstiger als im Westen, sodass selbst eine weitere Konvergenz des BIP je Erwerbstätigen, also der Arbeitsproduktivität, nicht zu einer Angleichung des BIP je Einwohner:in führen wird. Betrachtet man hingegen wichtige Wohlfahrtsindikatoren wie Konsummöglichkeiten, Lebenserwartung, Freizeit und Einkommensungleichheit, so sind die Lebensverhältnisse in Ost- und Westdeutschland ähnlicher als das BIP je Einwohner:in suggeriert. In den Debatten über den Auf- holprozess Ostdeutschlands sollte daher stärker auf die Arbeitsproduktivität als Maß für die Wirtschaftskraft und mehr auf andere Wohlfahrtsindikatoren als Maß für die Angleichung der Lebensverhältnisse abgestellt werden.

\section{Literatur}

Becker, S. O., L. Mergele und L. Woessmann (2020), The separation and reunification of Germany: Rethinking a natural experiment interpretation of the enduring effects of communism, Journal of Economic Perspectives, 34(2), 143-171.

BMWi (Bundesministerium für Wirtschaft und Energie) (2020), Jahresbericht der Bundesregierung zum Stand der Deutschen Einheit 2020.

Goebel, J., R. Habich und P. Krause (2009), Zur Angleichung von Einkommen und Lebensqualität im vereinigten Deutschland, Vierteljahreshefte zur Wirtschaftsforschung, 78(2), 122-145.

Holtemöller, O. und M. Irrek (2012), Wachstumsprojektion 2025 für die deutschen Länder: Produktion je Einwohner divergiert, Wirtschaft im Wandel, 18(4), 132-140.

Jones, C. I. und P. J. Klenow (2016), Beyond GDP? Welfare across countries and time, American Economic Review, 106(9), 2426-2457.

Lindner, A. (2017), Convergence between East German Regions and East-West Migration, in H.-G. Jeong und G. Heimpold (Hrsg.), Economic transition in unified Germany and implications for Korea, Korea Institute for International Economic Policy (KIEP) Policy References, 17-23, 133-148.

Roses, J. R. und N. Wolf (Hrsg.) (2019), The economic development of Europe's regions. A quantitative history since 1900, Routledge.

Statistisches Bundesamt (2019), Bevölkerungsentwicklung in den Bundesländern bis 2060. Ergebnisse der 14. Koordinierten Bevölkerungsvorausberechnung, Variante 2 (moderate Entwicklung der Geburtenhäufigkeit, Lebenserwartung und Wanderung).

Wieschemeyer, M. (2017), Internal migration in East Germany after Reunification: Demographic and Economic Effects, in H.-G. Jeong und G. Heimpold (Hrsg.): Economic transition in unified Germany and implications for Korea, Korea Institute for International Economic Policy (KIEP) Policy References, 17-23, 117-132.

Title: 30 Years after Reunification, Gross Domestic Product has Served its Purpose as an Indicator

Abstract: The comparison of living conditions in East and West Germany is often based on the gross domestic product per inhabitant. However, this measure is not a good welfare indicator in itself. It can be assumed that, measured by the gross domestic product per inhabitant, there will be no further significant equalisation of economic power in East and West Germany in the foreseeable future. This is because the age structure of East Germany, i.e. the ratio of employed persons to inhabitants, is less favourable than in the West. On the other hand, if one looks at important welfare indicators such as consumption opportunities, life expectancy, leisure time and income inequality, living conditions in East and West Germany are more similar than the gross domestic product per inhabitant suggests. In the debates on the catching-up process of East Germany, more emphasis should therefore be placed on labour productivity as a measure of economic strength and on welfare indicators as a measure of the equalisation of living conditions.

JEL Classification: I3, J1, O4, R1. 